\title{
Pus from the pylorus: an unusual endoscopic finding suggestive of periduodenal abscess
}

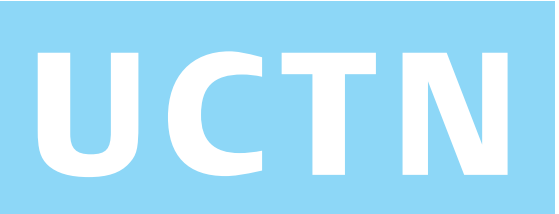

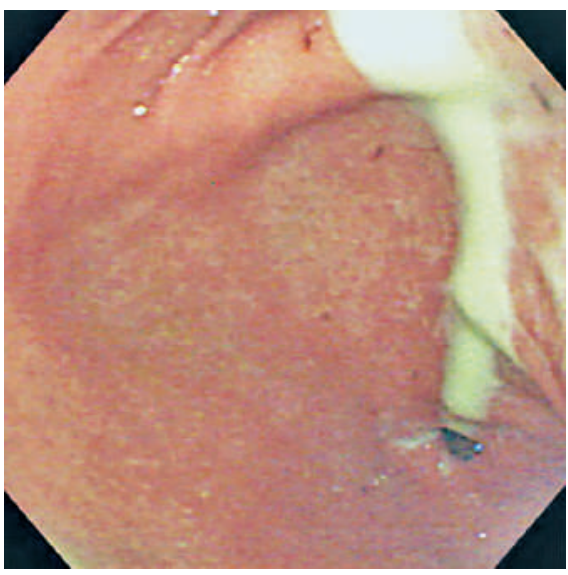

Figure 1 Endoscopic image, showing abundant whitish pus streaming from the pylorus into the gastric antrum.

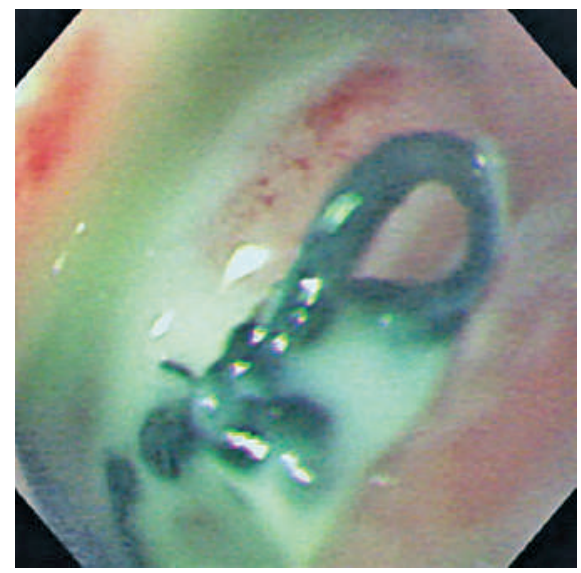

Figure 2 Endoscopic image, showing the pus originating from a sutured duodenal ulcer in the deformed duodenal bulb.

Subphrenic intra-abdominal abscess is a well-documented complication of perforated duodenal ulcers [1-5]. We report here on an unusual endoscopic finding of a sutured perforated duodenal ulcer associated with a subphrenic abscess.

A 32-year-old man in whom a perforated duodenal ulcer had been surgically closed with laparotomy 3 weeks previously presented to our emergency department with symptoms of persistent epigastralgia and fever. At esophagogastroduodenoscopy, abundant whitish pus was noted streaming from the pylorus into the gastric antrum (Figure $\mathbf{1}$ ). The source of the pus

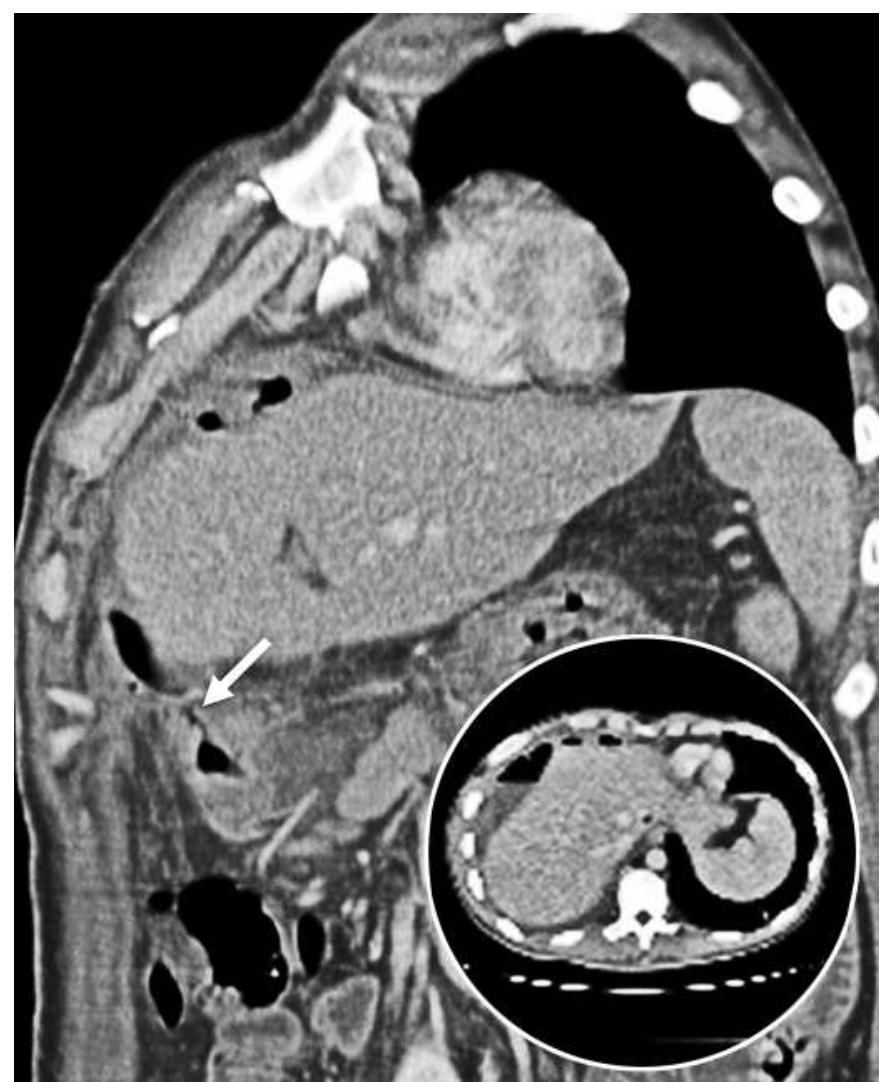

Figure 3 A reformatted computed tomogram, showing a fistula (arrow) communicating between the subphrenic abscess (see additional figure in the right lower corner) and the duodenal bulb.

was a large duodenal ulcer in the deformed duodenal bulb, where surgical sutures remained (Figure 2). A reformatted computed-tomographic image identified a fistula (Figure 3 ) between a subphrenic abscess and the duodenal bulb, accounting for the origin of the pus stream in the stomach. The patient received percutaneous drainage of the subphrenic abscess, parenteral antibiotics, and proton-pump inhibitors. Therapy to eradicate Helicobacter pylori was prescribed. He made an uneventful recovery without a repeat operation and remained well during a 6month follow-up period.

This case illustrates that a stream of pus in the stomach, particularly when it is accompanied by signs of infection, should suggest to endoscopists the possibility of a subphrenic abscess draining into the gastrointestinal tract.
Endoscopy_UCTN_Code_CCL-1AB-2AZ-3AC

C. C. Chen", T. C. Lee ${ }^{2}$, K. L. Liu ${ }^{3}$, J. T. Lin', H. P. Wang ${ }^{4}$

${ }^{1}$ Dept. of Internal Medicine, National Taiwan University Hospital Yun-Lin Branch, Yun-Lin, Taiwan

2 Dept. of Internal Medicine, National Taiwan University Hospital and National Taiwan University College of Medicine, Taipei, Taiwan

${ }^{3}$ Dept. of Medical Imaging

${ }^{4}$ Dept. of Emergency Medicine, National Taiwan University Hospital and National Taiwan University College of Medicine, Taipei, Taiwan. 


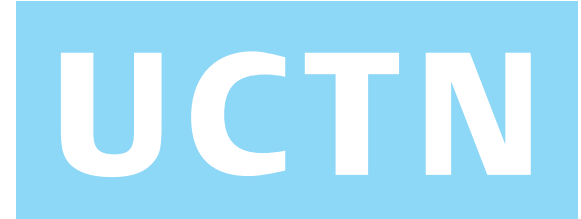

References

${ }^{1}$ Allard JC, Kuligowska E. Percutaneous treatment of an intrahepatic abscess caused by a penetrating duodenal ulcer. J Clin Gastroenterol 1987; 9: 603-606

${ }^{2}$ Albu E, Moreira D, Faltous A et al. Intramural abscess of the duodenum resulting from perforated peptic ulcer. South Med J 1995; 88: $1078-1080$

${ }^{3}$ Yoshida H, Onda M, Tajiri T et al. A case of abscess caused by a penetrating duodenal ulcer. Hepatogastroenterology 1999; 46: $2379-2381$
${ }^{4}$ Chau WK, Chan SC. Sonographic diagnosis of a small fistulous communication between a subphrenic abscess and a perforated duodenal ulcer. J Clin Ultrasound 2000; 28: 153 156

${ }^{5}$ Mimica M. Silent free perforation of duodenal ulcer in an elderly patient presenting with melena: management directed by upper endoscopy and percussion of the liver. Endoscopy 2001; 33: 387
Corresponding Author

\section{H. P. Wang, M.D.}

Dept. of Emergency Medicine

National Taiwan University Hospital and National Taiwan University College of Medicine

7 Chung-Shan South Road

Taipei 100

Taiwan

Fax: $\quad+886-2-23223150$

E-mail: wanghp@ntu.edu.tw 